\title{
Research Paper: Prognostic Factors of Aluminum Phosphide Poisoning in Urmia: A-five-years Cross-sectional Study
}

\author{
Mohammad Majidi' ${ }^{\text {(D) }}$ Mohammad Jamalpour² ${ }^{\text {D }}$, Solmaz Nekoueifard ${ }^{3 *}$ (D) \\ 1. Department of Forensic Medicine and Clinical Toxicology, School of Medicine, Urmia University of Medical Sciences, Urmia, Iran. \\ 2. Student Research Committee, School of Medicine, Urmia University of Medical Sciences, Urmia, Iran. \\ 3. Urmia Health Center, School of Medicine, Urmia University of Medical Sciences, Urmia, Iran.
}

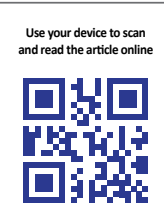

Citation Majidi M, Jamalpour M, Nekoueifard S. Prognostic factors of aluminum phosphide poisoning in Urmia: A-fiveyears Cross-sectional Study. International Journal of Medical Toxicology and Forensic Medicine. 2021; 11(2):32663. https://doi. org/10.32598/ijmtfm.v11i2.32663

doi https://doi.org/10.32598/ijmtfm.v11i2.32663

\section{(i) (\$)}

Article info:

Received: 23 Oct 2020

First Revision: 29 Oct 2020

Accepted: 18 Nov 2020

Published: 15 Jun 2021

\section{A B S T R A C T}

Background: Aluminum phosphide is a highly toxic fungicide. It causes such severe adverse effects as cardiac arrhythmias, metabolic acidosis, acute renal failure, shock, and even death. Therefore, this study investigated the prognostic factors of aluminum phosphide poisoning.

Methods: In this cross-sectional study, all hospitalized patients due to aluminum phosphide poisoning admitted to the poisoning center at Taleghani Hospital, Urmia City, Iran, from 2015 to 2019 were evaluated. The demographics characteristics, clinical findings, and laboratory profiles were retrospectively studied by an investigator-made checklist and evaluated concerning the explored patients' treatment outcomes. Then, the obtained data were analyzed by descriptive and analytical statistics using SPSS V. 16.

Results: The present study investigated 134 patients ( 96 males \& 38 females). The mortality rate was equal to $29.8 \%$ ( $22.4 \%$ males \& $7.4 \%$ females $)$ in the study patients. The research patients' Mean \pm SD age was $28.6 \pm 11.5$ ) years. The Mean \pm SD ingestion amount of aluminum phosphide was $1.48 \pm 1.06 \mathrm{~g}$ ( $\mathrm{min}=0.2 \mathrm{~g}$, $\max =15 \mathrm{~g})$. Nausea and vomiting with $119(88.8 \%)$, hypotension: 89 (66.4\%), vertigo: 80 (59.7\%), and sinus tachycardia: 74 (55.2\%) were the most signs and symptoms in the study patients, respectively. Statistically significant relationships $(\mathrm{P}<0.05)$ were found between the patients' treatment outcomes and white blood cells, direct bilirubin, $\mathrm{Hco}_{3}$ base excess, magnesium, Aspartate Aminotransferase, Alanine Aminotransferase, Blood Ưrea Nitrogen, creatinine, blood glucose, $\mathrm{pH}$, prothrombin time, and the international normalized ratio. Furthermore, significant relationships were detected between the patients' treatment outcomes and leukocytosis, hypokalemia, hyperglycemia, and hypoglycemia $(\mathrm{P}<0.05)$.

Conclusion: Such prognostic factors as demographics characteristics, clinical findings, laboratory profiles, and electrolytes could be used as good indices of the severity of toxicity in patients; accordingly, such data are beneficial for the proper management of patients by healthcare providers. Therefore, prognostic factors should be considered in the diagnosis, treatment, and follow-up stages for these patients.
Aluminum phosphide, Poisoning, Prognosis, Mortality 


\section{Introduction}

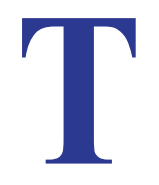

he rate of mortalities due to acute poisoning has increased worldwide [1]. Approximately, 300000 deaths due to pesticide poisoning occur in Asian pacific countries annually [2]. Aluminum Phosphide (ALP) poisoning usually occurs intentionally following suicidal ideation; in some cases, it occurs accidentally and rarely occurs criminally [3]. Considering its availability and low price, ALP is used to protect grains, like rice. As a result, it is also called the rice pill in Iran [4]. According to recent studies, suicide with rice pills has become highly prevalent in Iran [5]. ALP is a common cause of acute poisoning and mortality in numerous developing countries, like Iran $[1,6]$. ALP falls in the category of fungicides and rodenticides, i.e., available in the form of 3-gram tablets in dark gray with a smell similar to garlic or rotten fish $[6,7]$. The average lethal dose of ALP for an adult is $0.5 \mathrm{~g}$ or one-sixth of a pill $[3,8]$. The main routes of the involvement of ALP poisoning are oral administration and inhalation [6]. Rice pill, when combined with water or hydrochloric acid in the stomach, releases a highly toxic gas, called Phosphine (PH3). Phosphine gas acts by inhibiting the mitochondrial cytochrome oxidase system [5, 6]. The mortality rates of ALP intoxication were reported by Beyranvand et al. to range from $31 \%$ to $77 \%$ [9], Taghaddosinejad et al. as $70 \%-100 \%$ [10], and Navabi et al. as $60 \%-90 \%$ [3]. Due to high mortality, the production and distribution of rice tablets have been stopped since 2007 in Iran [1]. Multi organs failure, such as gastrointestinal, cardiovascular, hepatic, renal, and neurological involvement is observed in ALP poisoning due to its hypoxic effects $[5,6]$.

The important clinical manifestations of ALP poisoning are gastrointestinal conditions (nausea, vomiting, \& epigastric pain), cardiovascular disorders (hypotension \& even shock, tachycardia, bradycardia, \& congestive heart failure), central nervous system conditions (headache, dizziness, \& finally the loss of consciousness), and severe metabolic acidosis $[5,11]$. Life-threatening cardiac arrhythmias induced by severe metabolic acidosis are the main causes of death in ALP poisoning [3]. The diagnosis of ALP poisoning is usually based on clinical suspicions; however, silver nitrate testing on stomach contents or breathing vapors is also used to confirm the diagnosis [11]. ALP poisoning presents no specific antidote; however, previous studies have suggested some treatment approaches, such as gastric lavage with sodium bicarbonate and the ingestion of charcoal [12]. Additionally, some specific treatment methods of ALP poisoning have been suggested, including the intravenous administration of magnesium sulfate, sodium bicarbonate, $\mathrm{N}$-acetyl cysteine, digoxin, vasopressor, vitamin $\mathrm{C}$, and vitamin $\mathrm{E}[10$,
13, 14]. Some studies demonstrated that hyperglycemia, hypotension, acidosis, leukocytosis, hyperuricemia, electrocardiographic abnormalities, the loss of conciseness or coma, low prothrombin rate, and acute renal failure were associated with a poor prognosis in ALP poisoning $[3,4,7]$. Accordingly, laboratory profiles and their effects on the prognosis of patients with ALP poisoning were reported in limited studies [15-17]. Therefore, the present study evaluated demographics characteristics, clinical features, laboratory profiles, and their correlations with prognosis in ALP intoxicated patients.

\section{Materials and Methods}

The current study investigated 134 patients hospitalized due to ALP poisoning. They were admitted to the poisoning center at Taleghani Hospital in Urmia City, Iran from 2015 to 2019. To conduct this cross-sectional study, we included all patients with ALP poisoning in the age range of 14 years or above. In each case, the diagnosis had been made based on history gathered from the patients or their relatives. The hospital's clinical toxicologists also had visited the patients and provided further information to establish the diagnosis on admission to the Emergency Department (ED). Besides, the diagnosis of ALP exposure was made based on clinical signs and symptoms, such as inhaling garlic or rotten fish odor during their clinical examinations. ALP poisoning has no effective antidote; thus, the patient's management was only supportive. For all cases, gastric lavage with sodium bicarbonate, potassium permanganate, and activated charcoal were administered. Other components of administered supportive medical care included sodium bicarbonate, magnesium sulfate, calcium gluconate, Nacetyl cysteine, dopamine, and norepinephrine. Patients with incomplete records and laboratory tests during admission and those who were discharged with personal consent were excluded from this study.

Table 1 presents the study patients' demographics characteristics and clinical features. All laboratory profiles were checked upon hospital admission. Laboratory profiles and prognosis were retrospectively collected by an investigator-made checklist, i.e., presented in Table 2. Moreover, the comparison of mortality rate in studied patients from 2015 to 2019 are presented in Table 3. No personal identification data were recorded and all information remained strictly confidential. Approval for performing this research was issued by the Ethics Committee of Urmia University of Medical Sciences, Iran. The obtained data were analyzed in SPSS version 16 using descriptive statists, including Mean \pm SD. The data consisted of the demographic characteristics and clinical out- 
comes for every patient. The research variables were also grouped into survivors and nonsurvivors. In our study, the differences in quantitative variables with normal distributions and abnormal distributions were evaluated by the t-test and Mann-Whitney $U$ test, respectively. The relationships between categorical variables and the relevant outcomes were evaluated using Chi-squared test. Furthermore, $\mathrm{P}<0.05$ and confidence interval of $95 \%$ were found to be statistically significant in this research.

\section{Results}

The present study explored 134 patients (96 males \& 38 females). The mortality rate was calculated as $29.8 \%$
(22.4\% males \& 7.4\% females). The patients' Mean \pm SD age was measured as $28.6 \pm 11.5(\min =14$, $\max =81)$ years. The Mean \pm SD ingestion amount of ALP was equal to $1.48 \pm 1.06 \mathrm{~g}(\min =0.2 \mathrm{~g} \mathrm{max}=15 \mathrm{~g})$. The male to female ratio was calculated as 2:5. Table 1 presents the study patients' demographic characteristics and clinical features. The highest prevalence of aluminum phosphide poisoning $(67.9 \%)$ was reported in the age group of $14-30$ years. Approximately $12(9 \%)$ of patients presented no history of substance abuse. Furthermore, $17(12.5 \%)$ of the explored patients were rural and $117(87.5 \%)$ of them were urban residents. About 63 (47\%) of the investigated patients were married. Moreover, 95 (71\%) of cases reported a history of suicidal attempts. Nausea and vomiting 119 (88.8\%), hypo-

Table 1. Effects of some demographic characteristics and clinical features on the outcomes of the patients with ALP poisoning at admission

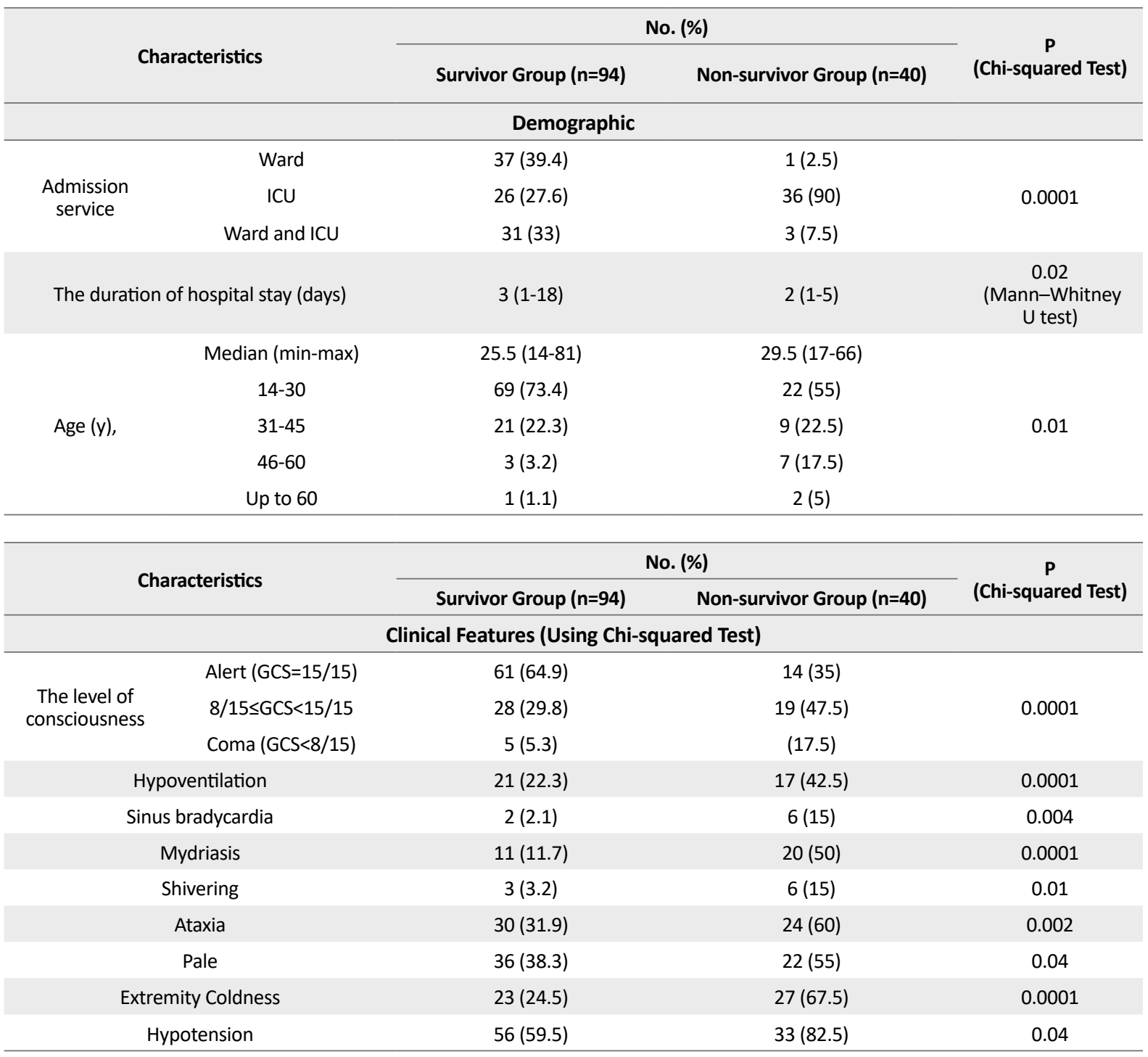

SD: Standard Deviation; GCS: Glasgow Coma Score; ARF: Acute Renal Failure. 
Table 2. Effects of some laboratory profiles on outcome and P-value at admission

\begin{tabular}{|c|c|c|c|c|}
\hline & \multirow{2}{*}{ Quantitative Variables } & \multicolumn{2}{|c|}{ Mean \pm SD/ No. (\%) } & \multirow{2}{*}{$\mathbf{P}$} \\
\hline & & Survivor Group ( $n=94)$ & Non-survivor Group ( $n=40)$ & \\
\hline \multirow{10}{*}{ 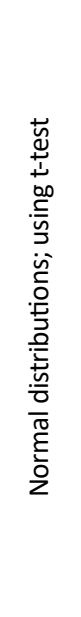 } & WBC (×103/L) & $9.5 \pm 4.1$ & $12.8 \pm 7.0$ & 0.000 \\
\hline & Direct Bilirubin (mg/dL) & $0.35 \pm 0.26$ & $0.6 \pm 0.1$ & 0.001 \\
\hline & Serum $\mathrm{HcO}_{3}$ & $21.7 \pm 5.9$ & $14.0 \pm 5.15$ & 0.000 \\
\hline & Base excess $(B E)(\mathrm{meq} / \mathrm{L})$ & $(-3) \pm(+6.3)$ & $(-14) \pm(-7.7)$ & 0.000 \\
\hline & $\mathrm{Hb}(\mathrm{mg} / \mathrm{dL})$ & $14.1 \pm 2.1$ & $14.2 \pm 1.7$ & 0.93 \\
\hline & HCT (\%) & $41.7 \pm 5.2$ & $42.0 \pm 7.3$ & 0.49 \\
\hline & PLT $(\times 103 / L)$ & $229.0 \pm 68.5$ & $212.0 \pm 63.0$ & 0.16 \\
\hline & Calcium (mg/dl) & $9.3 \pm 0.7$ & $9.0 \pm 0.6$ & 0.22 \\
\hline & Total Bilirubin (mg/dL) & $1.0 \pm 0.55$ & $1.2 \pm 0.2$ & 0.25 \\
\hline & $\mathrm{PcO}_{2}(\%)$ & $36.6 \pm 9.9$ & $34.9 \pm 13.5$ & 0.42 \\
\hline \multirow{14}{*}{ 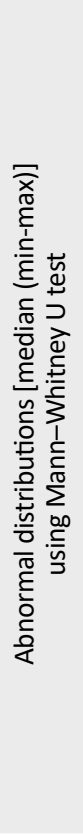 } & Magnesium (mg/dL) & $1.9(1.3-9.4)$ & $2.3(1.4-13)$ & 0.0001 \\
\hline & SGOT (IU/L) & $19(11-2465)$ & $54.5(16-218)$ & 0.007 \\
\hline & SGPT (IU/L) & $14(7-2328)$ & $50.5(13-174)$ & 0.006 \\
\hline & BUN (meq/L) & $22(11-53)$ & $28(16-71)$ & 0.0001 \\
\hline & Creatinine (meq/L) & $0.9(0.6-2.7)$ & $1.2(0.7-2.1)$ & 0.0001 \\
\hline & Blood Glucose (mg/dL) & $111(56-460)$ & $148(25-384)$ & 0.003 \\
\hline & Blood pH & $7.38(7.06-7.55)$ & $7.21(6.8-7.5)$ & 0.0001 \\
\hline & PT (s) & $13.2(11-20)$ & $14.7(12-60)$ & 0.001 \\
\hline & INR & $1.1(0.88-2.6)$ & $1.4(1-10.48)$ & 0.001 \\
\hline & Phosphor (mg/dL) & $3.2(1.2-8.2)$ & $5.8(1.9-3.9)$ & 0.053 \\
\hline & ALKP (IU/L) & $171(84-411)$ & $218(141-262)$ & 0.45 \\
\hline & Amylase (U/L) & $71(30-381)$ & $135(52-490)$ & 0.10 \\
\hline & Lipase (U/L) & $30(16-214)$ & $30(26-54)$ & 0.78 \\
\hline & $\operatorname{PTT}(s)$ & $30.3(25-180)$ & $31.5(23-125)$ & 0.118 \\
\hline \multirow{9}{*}{ 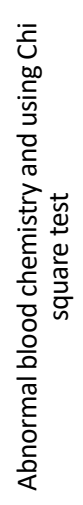 } & Leukocytosis (WBC>11000 / $\mathrm{mm}_{3}$ ) & $2(24.5)$ & $18(45)$ & 0.009 \\
\hline & Hypokalemia (K<3.5 meq/L) & $9(9.5)$ & $11(27.5)$ & 0.03 \\
\hline & Hyperglycemia (BG>200 mg/dL) & $7(7.4)$ & $12(30)$ & 0.02 \\
\hline & Hypoglycemia (BG<55 mg/dL) & 0 & $3(7.5)$ & 0.008 \\
\hline & Leukopenia (WBC<4500/mm3) & $6(6.4)$ & $1(2.5)$ & 0.62 \\
\hline & Thrombocytopenia (PLT<150000/L) & $10(10.6)$ & $2(5)$ & 0.45 \\
\hline & Hypernatremia ( $\mathrm{Na}>145 \mathrm{meq} / \mathrm{L})$ & 0 & $4(10)$ & 0.41 \\
\hline & Hyponatremia ( $\mathrm{Na}<135 \mathrm{meq} / \mathrm{L})$ & $2(2.1)$ & 0 & 0.19 \\
\hline & Hyperkalemia (K>5 meq/L) & $1(1.1)$ & $2(5)$ & 0.13 \\
\hline
\end{tabular}


Table 3. Comparing mortality rate in the survived and non-survived cases from 2015 to 2019

\begin{tabular}{|c|c|c|c|c|c|c|}
\hline \multirow{3}{*}{$\begin{array}{c}\text { Year of } \\
\text { Admission }\end{array}$} & \multicolumn{4}{|c|}{ No. } & \multirow{3}{*}{$\begin{array}{c}\text { Total } \\
(n=134)\end{array}$} & \multirow{3}{*}{$\begin{array}{c}\text { Mortality } \\
\text { Rate (\%) }\end{array}$} \\
\hline & \multicolumn{2}{|c|}{ Survivor Group ( $n=94)$} & \multicolumn{2}{|c|}{ Non-survivor Group $(n=40)$} & & \\
\hline & Male & Female & Male & Female & & \\
\hline 2015 & 1 & 0 & 2 & 0 & 3 & 66.6 \\
\hline 2016 & 13 & 3 & 7 & 2 & 25 & 36 \\
\hline 2017 & 11 & 4 & 4 & 0 & 19 & 21 \\
\hline 2018 & 22 & 10 & 8 & 6 & 46 & 30.4 \\
\hline 2019 & 19 & 11 & 9 & 2 & 41 & 26.8 \\
\hline
\end{tabular}

Table 4. Mortality and prognostic factors in our study, compared to some previous studies

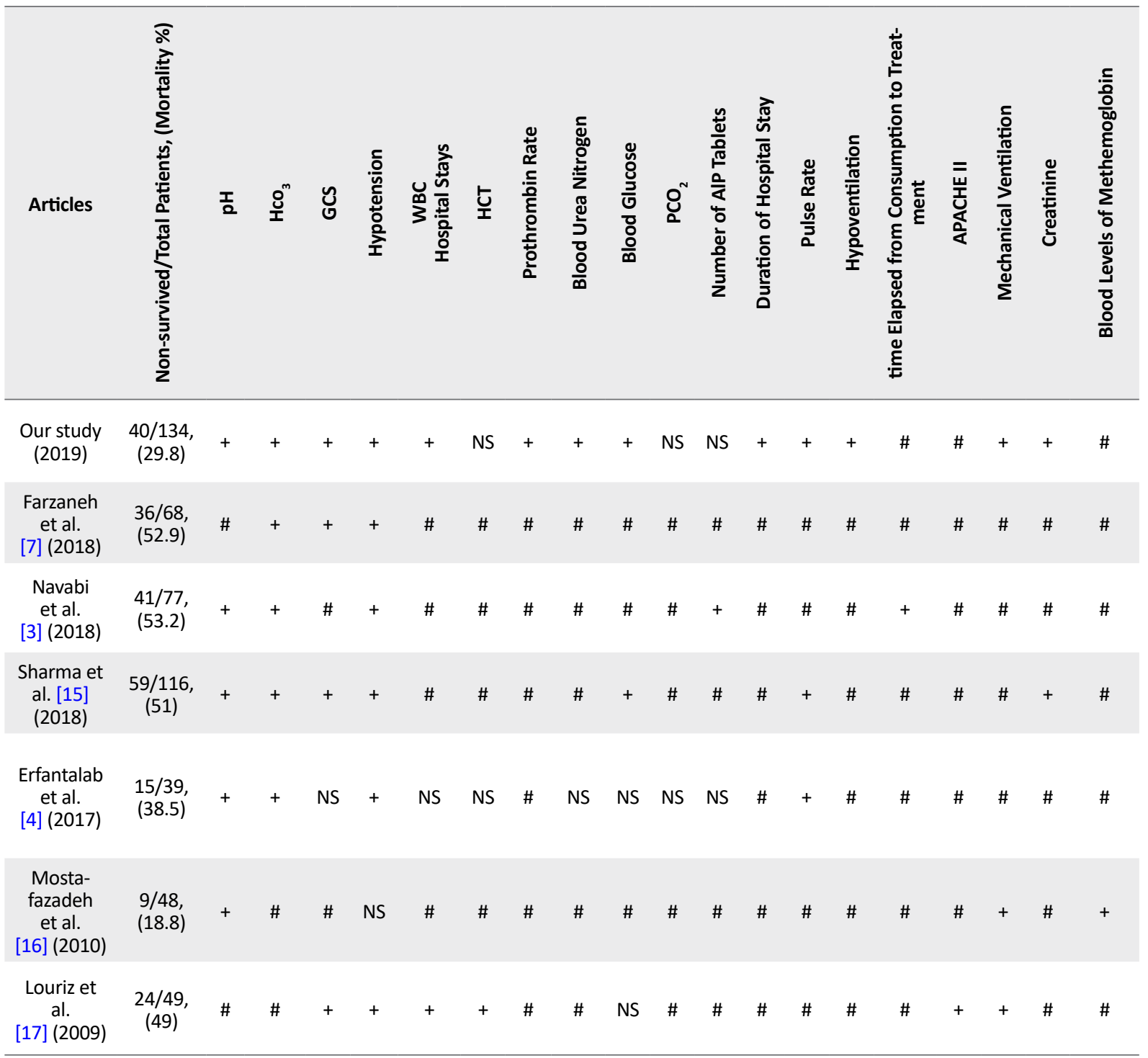

NS= Not Significant $=$ notperformed; $\mathrm{P}<0.05$ 
tension $89(66.4 \%)$, vertigo $80(59.7 \%)$, sinus tachycardia $74(55.2 \%)$, respiratory failure $73(54.5 \%)$, epigastric pain $70(52.2 \%)$, the loss of consciousness $65(48.5 \%)$, paler 58 (43.3\%), agitation $58(43.3 \%)$, ataxia $54(40.3 \%)$, extremity coldness $50(37.3 \%)$, mydriasis $31(23.1 \%)$, the dryness of mucosa $31(23.1 \%)$, tachypnea $27(20.1 \%)$, shivering 9 $(6.7 \%)$, miosis $9(6.7 \%)$, diarrhea $9(6.7 \%)$, and sinus bradycardia $8(6 \%)$ were the most prevalent signs and symptoms in the study patients, respectively. The results of the blood chemistry analyses range of the study patients at admission are presented in Table 2. Furthermore, 41 (30.6\%), $4(2.9 \%), 3(2.2 \%)$, and $19(14.2 \%)$ of the explored patients had leukocytosis, hypernatremia, hyperkalemia, and hyperglycemia, respectively. Additionally, 7 (5.2\%), 2 (1.45\%), $20(14.9 \%)$, and $3(2.2 \%)$ of the explored patients presented leukopenia, hyponatremia, hypokalemia, and hypoglycemia, respectively. The deceased patients, compared to the recovered ones (95\% vs. $51.5 \%$ ) swallowed $\geq 3$ grams of rice pills. Statistically significant relationships $(\mathrm{P}<0.05)$ were found between the study patients' treatment outcomes and demographic (admission service $\&$ the duration of hospital stay), clinical features (the level of consciousness, hypoventilation, sinus bradycardia, mydriasis, shivering, ataxia, pale, \& cold extremities) and laboratory profile, such as White Blood Cells (WBC), direct bilirubin, $\mathrm{Hco}_{3}$, base excess, magnesium, aspartate transaminase (SGOT), alanine aminotransferase (SGPT), Blood Urea Nitrogen (BUN), creatinine, blood glucose, $\mathrm{PH}$, Prothrombin Time (PT), and International Normalized Ratio (INR). Moreover, statistically significant relationships were observed between the study patients' treatment outcomes and leukocytosis, hypokalemia, hyperglycemia, and hypoglycemia (Table 2). However, there was no significant relationship between the study patients' treatment outcomes and demographic characteristics (history of substance abuse, living area, marital status, a history of suicidal attempts, and the amount of ingested ALP), clinical features (vomiting, nausea, agitation, vertigo, diarrhea, sinus tachycardia, epigastric pain, \& the dryness of mucosa), and laboratory profile, such as Hemoglobin (Hb), Hematocrit (HCT), Platelets (PLT), Partial Thromboplastin time (PTT), calcium, phosphor, total bilirubin, Alkaline Phosphatase (ALKP), amylase, lipase, and partial pressure of carbon dioxide (Pco2).

\section{Discussion}

ALP is a highly-toxic pesticide with a high mortality rate. The mean age of the study cases was 28.6 years. Overall, the consequences of this investigation are compatible with recent studies, such as Erfantalab et al., Kordrostami et al., and Mostafazadeh et al., i.e., 31, 32.6, and 25.5 years respectively. These studies indi- cated that ALP poisoning has increased among young individuals and the majority of the patients were male in Iran $[4,16,18]$. The highest prevalence of ALP poisoning $(67.9 \%)$ belonged to the age group of 14-30 years. All explored patients were poisoned following suicidal attempts, i.e., similar to the study of Navabi et al. [3] as well as Hosseinian and associates [19]. In this study, the number of ingested ALP on admission were different in the survivor group, compared to the non-survivor group. Additionally, these data provided useful information; however, consistent with the study of Erfantalab et al., they had no prognostic value in ALP intoxicated patients [4]. In line with the recent studies, the early clinical findings at admission were gastrointestinal discomforts, such as nausea, vomiting, and abdominal pain [19, 20]. Consistent with the study of Jamshidi et al., approximately $15 \%, 2 \%$, and $2 \%$ of the investigated patients reported hypokalemia, hyperkalemia, and hyponatremia, respectively [1]. Concerning Arterial Blood Gases (ABG), serum $\mathrm{Hco}_{3}$ and $\mathrm{pH}$ levels were very low in the non-surviving group, compared to the surviving group. Such finding is consistent with those of other studies [1, 15]. Interestingly, similar to the results of Sharma et al., the mean blood glucose level in this study was very low in the surviving group, compared to the non-surviving group [15]. Most studies have reported mortality rates of above $50 \%$; however, in this study, the mortality rate due to ALP poisoning was measured as $29.8 \%$, i.e., less than those reported by most previous studies. Approximately, the mortality rate of this study is consistent with those of Erfantalab et al. [4] and Mostafazadeh et al. [16] (Table 4). ALP poisoning has no specific antidote; thus, its management is supportive. Therefore, early diagnosis using appropriate predictors of mortality may provide desirable care and prognosis for these patients. Numerous prognostic factors for ALP poisoning have been used to evaluate the severity of patient's clinical conditions, such as blood $\mathrm{pH}$ (severe acidosis), serum $\mathrm{Hco}_{3}$, hyperglycemia, GCS, and blood pressure, age, the lack of vomiting, dose ingested APACHE II, white blood cell count, mechanical ventilation, creatinine, blood urea nitrogen, blood levels of methemoglobin, hospital stays, hematocrit, prothrombin rate, $\mathrm{PCO} 2$, the number of ALP tablets, the duration of hospital stay, blood urea nitrogen, blood glucose, pulse rate, hypoventilation, and time elapsed from consumption to treatment (Table 4) $[3,7,15-17]$. Furthermore, our study demonstrated that magnesium, aspartate transaminase, alanine aminotransferase, International Normalized Ratio (INR), direct bilirubin, mydriasis, shivering, ataxia, pale, and cold extremities were significantly different between survivors and non-survivors groups. Finally, to obtain 
better results, follow-up studies are recommended to investigate the other prognostic factors.

The main limitation of this study was that the diagnosis of ALP poisoning was not confirmed by the silver nitrate test. Another limitation of this study was the incomplete recording of some laboratory data.

\section{Conclusion}

Laboratory abnormalities, such as severe metabolic acidosis, clinical suspicion (severe hypotension), and a positive history of ALP poisoning can be applied for early diagnosis and appropriate treatment of this intoxication. The amount of ingested ALP on admission provides useful information; however, these data presented no prognostic value in patients with ALP poisoning. Establishing the relevant prognostic factors in the clinical setting is crucial for managing patients with acute ALP poisoning. Previous research studies addressed some prognostic factors and their effects on the prognosis of ALP poisoning outcomes. Furthermore, prognostic factors should be considered in the diagnosis, treatment, and follow-up stage of these patients. However, some prognostic factors, such as laboratory profiles, electrolytes, and clinical findings could be used as appropriate indices of the severity of the toxicity of the patients; these data can be used for proper management by healthcare providers. Further studies are required to elucidate definitive prognostic factors in patients with ALP poisoning.

\section{Ethical Considerations}

\section{Compliance with ethical guidelines}

The Ethics Committee of Urmia University of Medical Sciences approved this study (Code: IR.UMSU.REC.1399.215).

\section{Funding}

This article was supported by the Urmia University of Medical Science.

\section{Author's contributions}

All authors contributed to preparing this article.

\section{Conflict of interest}

The authors declared no conflicts of interest.

\section{Acknowledgements}

The authors would like to thank the management and staff of Taleghani Hospitals, Urmia, for approving its protocol prior to implementation and support this study.

\section{References}

[1] Jamshidi F, Ghorbani A, Darvishi S, Davoodzadeh H. Study of laboratory profile in patients with aluminium phosphide poisoning in the southwest of Iran from 2010 to 2015. Arch Med Sadowej Kryminol. 2016; 66(3):149-57. [Doi:10.5114/amsik.2016.66399] [PMID]

[2] Hassanian-Moghaddam H, Zamani N. Therapeutic role of hyperinsulinemia/euglycemia in aluminum phosphide poisoning. Medicine (Baltimore). 2016; 95(31):e4349. [Doi:10.1097/ MD.0000000000004349] [PMID][PMCID]

[3] Navabi SM, Navabi J, Aghaei A, Shaahmadi Z, Heydari R. Mortality from aluminum phosphide poisoning in Kermanshah Province, Iran: Characteristics and predictive factors. Epidemiol Health. 2018; 40:e2018022. [Doi:10.4178/epih.e2018022] [PMID] [PMCID]

[4] Erfantalab P, Soltaninejad K, Shadnia S, Zamani N, HassanianMoghaddam H, Mahdavinejad A, et al. Trend of blood lactate level in acute aluminum phosphide poisoning. World J Emerg Med. 2017; 8(2):116-20. [Doi:10.5847/wjem.j.1920-8642.2017.02.006] [PMID] [PMCID]

[5] Shadnia S, Sasanian G, Allami P, Hosseini A, Ranjbar A, AminiShirazi N, et al. Aretrospective 7-years study of aluminum phosphide poisoning in Tehran: Opportunities for prevention. Hum Exp Toxicol. 2009; 28(4):209-13. [Doi:10.1177/0960327108097194] [PMID]

[6] Etemadi-Aleagha A, Akhgari M, Iravani FS. Aluminum phosphide poisoning-related deaths in Tehran, Iran, 2006 to 2013. Medicine (Baltimore). 2015; 94(38):e1637. [Doi: 10.1097/ MD.0000000000001637] [PMID] [PMCID]

[7] Farzaneh E, Ghobadi H, Akbarifard M, Nakhaee S, Amirabadizadeh A, Akhavanakbari G, et al. Prognostic factors in acute aluminium phosphide poisoning: A risk-prediction nomogram approach. Basic Clin Pharmacol Toxicol. 2018; 123(3):347-55. [Doi:10.1111/bcpt.13005] [PMID]

[8] Olson KR, Anderson IB, Benowitz NL, Blanc PD, Clark RF Kearney TE, et al. Poisoning \& drug overdose. $7^{\text {th }}$ ed. New York: McGraw-Hill Education; 2017. https:/ / accessmedicine.mhmedical.com/book.aspx?bookID=2284

[9] Beyranvand MR, Farrokhi S, Peyvandi H, Soltaninejad K, Shadnia SH. The effects of amiodarone prophylaxis on cardiac dysrhythmia in acute aluminium phosphide poisoning. Arh Hig Rada Toksikol. 2019; 70(1):49-53. [Doi:10.2478/aiht-2019-70-3162] [PMID]

[10] Taghaddosinejad F, Farzaneh E, Ghazanfari-Nasrabad M, Eizadi-Mood N, Hajihosseini M, Mehrpour O. The effect of NAcetyl Cysteine (NAC) on aluminum phosphide poisoning inducing cardiovascular toxicity: A case-control study. Springerplus. 2016; 5(1):1948. [Doi:10.1186/s40064-016-3630-2] [PMID] [PMCID]

[11] Mashayekhian M, Hassanian Moghaddam H, Rahimi M Zamani N, Aghabiklooei A, Shadnia S. Elevated carboxyhaemoglobin concentrations by pulse co-oximetry is associated with 
severe aluminium phosphide poisoning. Basic Clin Pharmacol Toxicol. 2016; 119(3):322-9. [Doi:10.1111/bcpt.12571] [PMID]

[12] Maleki A, Hosseini MJ, Rahimi N, Abdollahi A, Akbarfakhrabadi A, Javadian N, et al. Adjuvant potential of selegiline in treating acute toxicity of aluminium phosphide in rats. Basic Clin Pharmacol Toxicol. 2019; 125(1):62-74. [Doi:10.1111/ bcpt.13207] [PMID]

[13] Tehrani H, Halvaie Z, Shadnia S, Soltaninejad K, Abdollahi M. Protective effects of $\mathrm{N}$-acetylcysteine on aluminum phosphideinduced oxidative stress in acute human poisoning. Clin Toxicol (Phila). 2013; 51(1):23-8. [Doi:10.3109/15563650.2012.743029 ] [PMID]

[14] Karimani A, Mohammadpour AH, Zirak MR, Rezaee R, Megarbane B, Tsatsakis A, et al. Antidotes for aluminum phosphide poisoning - An update. Toxicol Rep. 2018; 5:1053-9. [Doi: 10.1016/j.toxrep.2018.10.009] [PMID] [PMCID]

[15] Sharma A, Balasubramanian P, Gill KD, Bhalla A. Prognostic significance of blood glucose levels and alterations among patients with aluminium phosphide poisoning. Sultan Qaboos Univ Med J. 2018; 18(3):e299-303. [Doi:10.18295/squmj.2018.18.03.006] [PMID] [PMCID]

[16] Mostafazadeh B, Pajoumand A, Farzaneh E, Aghabiklooei A, Rasouli MR. Blood levels of methemoglobin in patients with aluminum phosphide poisoning and its correlation with patient's outcome. J Med Toxicol. 2011; 7(1):40-3. [Doi:10.1007/s13181010-0121-7] [PMID] [PMCID]

[17] Louriz M, Dendane T, Abidi K, Madani N, Abouqal $\mathrm{R}$, Zeggwagh AA. Prognostic factors of acute aluminum phosphide poisoning. Indian J Med Sci. 2009; 63(6):227-34 [Doi:10.4103/0019-5359.53386] [PMID]

[18] Kordrostami R, Akhgari M, Ameri M, Ghadipasha M, Aghakhani K. Forensic toxicology analysis of self-poisoning suicidal deaths in Tehran, Iran; Trends between 20112015. Daru. 2017; 25(1):15. [Doi:10.1186/s40199-017-0181-1] [PMID] [PMCID]

[19] Hosseinian A, Pakravan N, Rafiei A, Feyzbakhsh SM. Aluminum phosphide poisoning known as rice tablet: A common toxicity in North Iran. Indian J Med Sci. 2011; 65(4):14350. [Doi:10.4103/0019-5359.104777] [PMID]

[20] Moghadamnia AA. An update on toxicology of aluminum phosphide. Daru. 2012; 20(1):25. [Doi:10.1186/2008-2231-2025] [PMID] [PMCID] 